\title{
Acute small bowel obstruction owing to undiagnosed lung cancer
}

\author{
Katerina Erokhina, DO, ${ }^{\mathrm{a}}$ Nazar Sharak, DO, ${ }^{\mathrm{b}}$ Wayne Cai, MD, ${ }^{\mathrm{c}}$ Berry Pierre, DO, ${ }^{\mathrm{a}}$ and \\ Allen Schultz, DO ${ }^{\mathrm{d}}$
}

${ }^{a}$ Department of Medicine, Palm Beach Consortium for Graduate Medical Education, West Palm Beach, Florida; ${ }^{b}$ Department of Medicine, Broward Health Medical Center, Fort Lauderdale, Florida; 'Department of Clinical Pathology, Palm Beach Consortium for Graduate Medical Education, West Palm Beach, Florida; and ${ }^{\mathrm{d} S o u t h}$ Florida Oncology, West Palm Beach, Florida

L

ung cancer is the leading cause of cancer related deaths in the United States. Most patients with lung cancer present with advanced disease at the time of diagnosis owing to multiple metastases. However, metastases to the duodenum are extremely rare. We present a case of acute small bowel obstruction in a patient owing to metastatic disease to the duodenum from previously undiagnosed lung adenocarcinoma.

\section{Case presentation}

A 49-year-old white woman presented to the emergency department because of intractable bilious vomiting for the duration of 1 month. The patient was previously admitted to another facility for small bowel obstruction, which resolved with conservative treatment. Shortly after she had been discharged, the patient developed nonbloody, bilious emesis on an almost daily basis for the last 3 weeks, with an associated 17-pound weight loss. The patient also reported to new-onset constipation and diffuse abdominal pain. She had an extensive history of smoking and alcoholism, but no pertinent past medical or surgical histories. Her use of home medications was limited to over-the-counter laxatives, which she had started to take recently for constipation, and ibuprofen for abdominal pain.

The results of a physical exam were significant for diffuse abdominal tenderness and hyperactive bowel sounds. A computed-tomography (CT) scan of abdomen demonstrated a high-grade small bowel obstruction (Figure 1A). Based on the radiology findings, we decided to hospitalize the patient and to insert a nasogastric tube. However, the patient failed the conservative treatment, and on the day 4 of hospitalization, she underwent an exploratory laparotomy. The surgical intervention visualized the obstruction of small intestine, along with a new infiltrating circumferential process of the small bowel extending from the stomach to the ligament of Treitz. About 12 inches of small bowel containing the invasive mass had to be resected out.

After the surgery, the patient remained on mechanical ventilator support because she experienced respiratory failure. On day 2 after the surgery, the patient's hemodynamic status began to deteriorate and vasopressors were initiated. By the end of the first week of hospitalization, the patient developed heparin-induced thrombocytopenia and hypercoagulable state, which led to ischemia of the right toes; multiple bilateral intracranial nonhemorrhagic infarctions, with the largest one in the right occipital lobe (Figure 1B); and bilateral pulmonary emboli (Figure 1C). CT angiography (CTA) of the chest also revealed 2 speculated lesions -1.3 $\mathrm{cm} \times 0.6 \mathrm{~cm}$ in the left upper lobe, and $1.0 \mathrm{~cm} \mathrm{x}$ $1.3 \mathrm{~cm}$ in the right upper lobe (Figure 1D) - extensive mediastinal lymphadenopathy with necrotic lymph nodes, superior vena cava narrowing owing to mass effect and left adrenal mass likely because of metastases. The patient was unable to tolerate direct thrombin inhibitors because of her gastrointestinal bleeding, so an inferior vena cava filter was inserted. In addition to all these events, the patient developed intermittent atrial fibrillation with pericardial effusion. Pericardial fluid obtained during pericardiocenthesis was later found to be of malignant etiology.

The size of the resected duodenum was about 12 inches. A histological analysis of the specimen revealed poorly differentiated, high-grade (grade III) adenocarcinoma (Figure $2 \mathrm{~A}$ and Figure 2B) with 6 out of 6 mesenteric lymph nodes positive for metastatic carcinoma. The tumor was transmural and involved the mucosa, the submucosa, the muscular bowel wall, and the serosa. Proximal and

Accepted for publication June 10, 2014. Correspondence: Katerina Erokhina, DO; Eerokhina@osteo.wvsom.edu. Disclosures: The authors have no disclosures. JCSO 2014;12:298-300. (2014 Frontline Medical Communications. DOI 10.12788/icso.0067 
distal resection margins were free of tumor. Furthermore, the tumor was positive for thyroid transcription factor-1 (TTF-1) and cytokeratin-7 (CK-7) and was negative for cytokeratin-20 (CK-20). Cells obtained from pericardial effusion (Figure 2C) stained strongly for MOC-31 monoclonal antibody and carcinoembryonic antigen

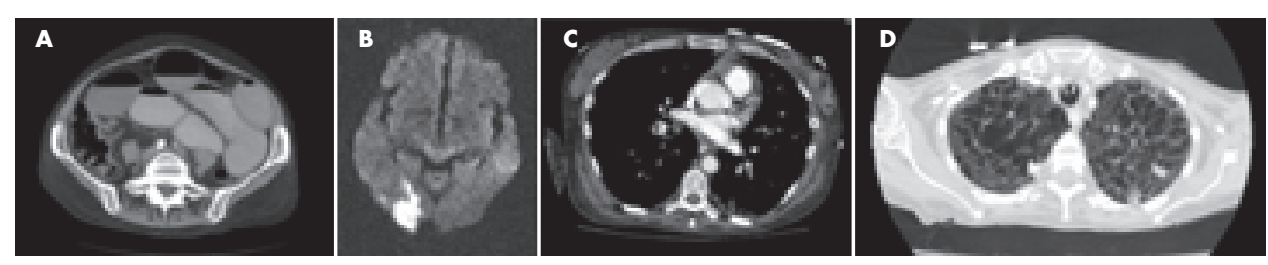

FIGURE 1 A, Computed-tomography imaging showing high-grade mechanical bowel obstruction. B, Axial DW imaging depicting stroke in right occipital lobe. C, CT imaging showing bilateral PE. D, CT imaging showing 2 pulmonary nodules in RUL and LUL

(CEA), in addition to being posi-

tive for TTF-1 and CK-7. These findings were consistent with primary adenocarcinoma of the lung.

On hospital day 37, patient could not be weaned off of the mechanical ventilator and required continuous vasopressor infusion. It was then that the family had decided to transfer the patient to hospice care.

\section{Discussion}

Lung cancer is the leading cause of cancer related deaths in the United States. Lung adenocarcinoma is the most common histological type of lung cancer in men and women. ${ }^{1}$ The main risk factor for lung cancer is smoking, because it leads to reprogramming of airway basal cell/progenitor cell population and activation of human embryonic stem cells with subsequent higher frequency of TP53 mutations. ${ }^{2}$ There are many other additional environmental and occupational risk factors, as well as genetic predispositions with several mutations identified. ${ }^{3}$ Patients with several of these factors have an increased risk of developing lung malignancy because of synergetic effect. ${ }^{4}$

About half of the patients with non-small-cell lung cancer present with metastatic disease. ${ }^{5}$ However, symptomatic bowel metastases from primary lung cancer are rare and usually occur after the diagnosis of lung cancer has been made. ${ }^{6,7}$ According to the study described by Nishizawa and colleagues, only $0.17 \%$ of 4,114 patients with lung cancer developed symptomatic small bowel metastases. ${ }^{8}$ However, the rate of gastrointestinal metastases found on autopsy of patients who have died of lung cancer is much higher - up to $14 \%$. The most likely explanation for this is that most metastases are asymptomatic. ${ }^{9}$ The most common site of gastrointestinal metastases in lung cancer patients is the esophagus, particularly, its middle portion, and the most common location of the intestinal metastases is jejunum. ${ }^{9,10}$ Metastases to the duodenum are exceedingly rare compared with metastases to other sites on the gastrointestinal tract. ${ }^{11}$

Although all histological types of cancer can metastasize to the small bowel, the most common type is squamous cell, followed by a large cell carcinoma. ${ }^{6,9,12}$ There is a significant male predominance in patients with small bowel metastases, and the median age of diagnosis in all patients is 60 years. ${ }^{9}$

Metastatic disease that invades the abdomen often manifests itself with pain. The most common presentation is perforation, owing to the high predisposition of lung cancers toward necrosis, ${ }^{12}$ whereas obstruction usually occurs as a consequence of rapid tumor growth. Other presentations of abdominal metastases include obstruction, intussusception, and gastrointestinal bleeding. ${ }^{13}$ It has also been shown that patients who have metastases to small bowel generally have a poor prognosis because they occur in the later stages of the disease. ${ }^{14}$

Patients with adenocarcinoma are prone to hypercoagulable state, which might lead sometimes to systemic ischemic events as described in this case. Usually, a hypercoagulable state is associated with a poor prognosis and a failure of systemic anticoagulants. Medical management of such cases includes the initiation of chemotherapy and treatment of underlying tumor. ${ }^{15,16}$

Immunohistochemistry is an important tool in the diagnosis of lung cancer. TTF- 1 marker is positive in about $70 \%$ of primary pulmonary adenocarcinoma, whereas metastatic disease to the lungs is usually TTF-1 negative. ${ }^{17}$ TTF- 1 is also positive in primary thyroid tumors; however, in the case of our patient, thyroglobulin was negative. The tumor stained positive for CK-7 and was negative for CK-20, which is consistent with primary pulmonary

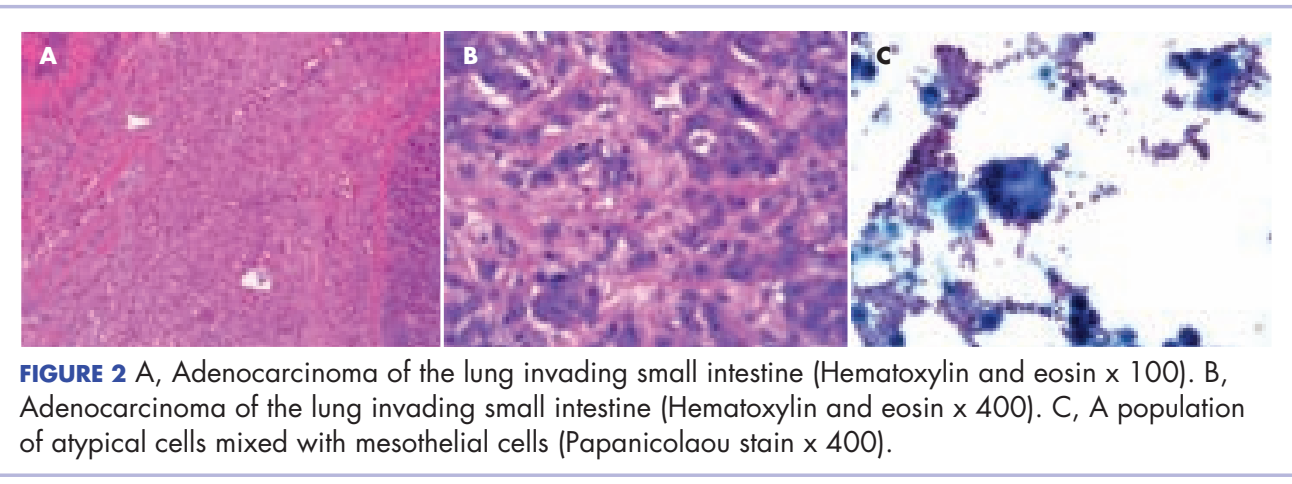


malignancy contrary to colorectal adenocarcinomas, which usually stain positive for CK-20 and negative for CK-7. ${ }^{18}$

Another unusual feature of this case was the magnitude of metastatic disease compared with the relatively small volume of the primary tumor. To date, a number of gross and molecular features of the tumor have been defined as risk factors favoring the distal metastasis. These include tumor size, lymph node spread, probability for angiogenesis, genetic structure of the tumor, and expression of different biomarkers. ${ }^{19}$ Overall, patients with a history of cigarette smoking have a higher risk of metastatic disease. ${ }^{20}$

\section{Conclusion}

We described a case of unusual and rapidly progressing presentation of small bowel obstruction secondary to a previously undiagnosed metastatic adenocarcinoma of the lung. Undeterred by sizable excision of metastatic mass within small bowel, the postoperative course was complicated by hypercoagulable state with embolic sequela of ischemia of toes, multifocal intracranial infarctions, and bilateral pulmonary emboli. Metastatic disease also spread to the pericardial cavity and caused a tamponade that required pericardiocenthesis. This case highlights rapidly progressing and lethal complications of metastatic lung adenocarcinoma and the various challenges the physicians might have to face in attempt to manage these complications.

\section{References}

1. Wahbah M, Boroumand N, Castro C, El-Zeky F, Eltorky M. Changing trends in the distribution of the histologic types of lung cancer: a review of 4,439 cases. Ann Diagn Pathol. 2007;11:89-96.

2. Shaykhiev R, Wang R, Zwick RK, et al. Airway basal cells of healthy smokers express an embryonic stem cell signature relevant to lung cancer. Stem Cells. 2013;31:1992-2002.

3. Clapp RW, Jacobs MM, Loechler EL. Environmental and occupational causes of cancer: new evidence 2005-2007. Rev Environ Health. 2008;23:1-37.

4. Le Calvez F, Mukeria A, Hunt JD, et al. TP53 and KRAS mutation load and types in lung cancers in relation to tobacco smoke: distinct patterns in never, former, and current smokers. Cancer Res. 2005;65:5076-5083.
5. Mohammed TL, Chowdhry A, Reddy GP, et al. ACR Appropriateness Criteria(R) screening for pulmonary metastases. J Thorac Imaging. 2011;26:W1-3.

6. Berger A, Cellier C, Daniel C, et al. Small bowel metastases from primary carcinoma of the lung: clinical findings and outcome. Am J Gastroenterol. 1999;94:1884-1887.

7. Sanli Y, Adalet I, Turkmen C, Kapran Y, Tamam M, Cantez S Small bowel metastases from primary carcinoma of the lung: presenting with gastrointestinal hemorrhage. Ann Nucl Med. 2005;19:161-163

8. Nishizawa Y, Kobayashi A, Saito N, et al. Surgical management of small bowel metastases from primary carcinoma of the lung. Surg Today. 2012;42:233-237.

9. Hillenbrand A, Strater J, Henne-Bruns D. Frequency, symptoms and outcome of intestinal metastases of bronchopulmonary cancer. Case report and review of the literature. Int Semin Surg Oncol.. 2005;2:13.

10. Antler AS, Ough Y, Pitchumoni CS, Davidian M, Thelmo W. Gastrointestinal metastases from malignant tumors of the lung. Cancer. 1982;49:170-172.

11. Yilmaz S, Dursun M, Canoruc F, Bayan K, Buyukbayram H. Upper gastrointestinal bleeding caused by small-cell lung cancer: a case report. Dig Dis Sci. 2006;51:788-790.

12. McNeill PM, Wagman LD, Neifeld JP. Small bowel metastases from primary carcinoma of the lung. Cancer. 15 1987;59:1486-1489.

13. Garwood RA, Sawyer MD, Ledesma EJ, Foley E, Claridge JA. A case and review of bowel perforation secondary to metastatic lung cancer.Am Surg . 2005;71:110-116.

14. Kostakou C, Khaldi L, Flossos A, Kapsoritakis AN, Potamianos SP Melena: a rare complication of duodenal metastases from primary carcinoma of the lung. World J Gastroenterol. 2007;13:1282-1285.

15. Park HY, Lee HS, Chang H, Kim YS, Cho KH. Multifocal strokes as the initial manifestation of metastatic tumors. Neurologist. 2010;16:322-324

16. Navi BB, DeAngelis LM, Segal AZ. Multifocal strokes as the presentation of occult lung cancer. J Neurooncol 2007;85:307-309.

17. Jagirdar J. Application of immunohistochemistry to the diagnosis of primary and metastatic carcinoma to the lung. Arch Pathol Lab Med. 2008;132:384-396.

18. Kummar S, Fogarasi M, Canova A, Mota A, Ciesielski T. Cytokeratin 7 and 20 staining for the diagnosis of lung and colorectal adenocarcinoma.Br J Cancer . 2002;86:1884-1887.

19. O’Byrne KJ, Koukourakis MI, Giatromanolaki A, et al. Vascular endothelial growth factor, platelet-derived endothelial cell growth factor and angiogenesis in non-small-cell lung cancer. $\mathrm{Br} \mathrm{J}$ Cancer . 2000;82:1427-1432.

20. Maeda R, Yoshida J, Ishii G, Hishida T, Nishimura M, Nagai K. Influence of cigarette smoking on survival and tumor invasiveness in clinical stage IA lung adenocarcinoma. Ann Thorac Surg . 2012;93:1626-1632. 